\title{
Die Ökonomik und ihre Schwierigkeiten mit der Moral
}

\author{
MARTIN KOLMAR UND THOMAS BESCHORNER ${ }^{* *}$
}

Vor dem Hintergrund einer kontroversen Diskussion auf der Plattform „Ökonomenstimme“ zu „Schwierigkeiten mit der Moral“ in der Wirtschaftspraxis diskutiert der vorliegende Beitrag Probleme der traditionellen Wirtschaftswissenschaften hinsichtlich der Konzeptualisierung moralischer Dimensionen in ökonomischen Theorien. Die Autoren zeigen, dass eine reine Anreizethik, die lediglich auf veränderte Spielregeln abstellt, in theoretischer Hinsicht nicht plausibilisiert werden kann. Moralisches Handeln ist stattdessen als ein Handlungstypus sui generis zu verstehen und das Wechselspiel von moralischem Handeln und Institutionen ist in den Mittelpunkt der Untersuchung zu rücken. Aus diesen Perspektiven ergeben sich durchaus weitreichende ontologische und epistemologische Grundsatzfragen für die Ökonomik.

Schlagwörter: Wirtschaftswissenschaften, Moral, Ethik, Institutionen, Handeln, Wissenschaftstheorie

\section{Economics and its Difficulties with Morality}

This paper discusses mainstream economics and its problems with conceptualizing individual moral responsibility within its theoretical framework. It refers to a controversial debate hosted at the online platform 'Ökonomenstimme' and our article on 'Difficulties with Morality'. We argue that the methodological approach, to exclusively rely on the design of institutions to solve ethical problems, rests upon anthropological and epistemic premises that are highly problematic and difficult to defend. Instead, we need to understand moral action as a distinct type of action and therefore to shift our focus towards studying the relationship between moral action and institutions. This perspective has potentially farreaching implications for the way we conceptualize the relationship between individual, society, and state.

Keywords: Economics, Morals, Etbics, Institutions, Action, Pbilosophy of Science

Beitrag eingereicht am 05.11.2015, es handelt sich um eine leicht erweiterte Fassung des Beitrages auf „Ökonomenstimme“: Beschorner/Kolmar (2015a).

Prof. Dr. Martin Kolmar, Forschungsgemeinschaft für Nationalökonomie, Universität St. Gallen, Varnbüelstr. 19, CH-9000 St. Gallen, Tel.: +41-(0)71-224 2535, Fax: +41-(0)71-224 2302, EMail: martin.kolmar@unisg.ch, Forschungsschwerpunkte: Konflikttheorie, Institutionenökonomik, normative Ökonomik, Verhaltenstheorie

Prof. Dr. Thomas Beschorner, Institut für Wirtschaftsethik, Universität St. Gallen, Girtannerstr. 8, CH-9010 St. Gallen, Tel.: +41-(0)71-224 3143, Fax: +41-(0)71-224 2881, E-Mail: thomas.beschorner@unisg.ch, Forschungsschwerpunkte: Wirtschafts- und Unternehmensethik, Handlungs- und Institutionentheorie. 


\section{Hintergrund}

Unsere Beiträge „Schwierigkeiten mit der Moral: Ein Kommentar aus gegebenem Anlass“ (Beschorner/Kolmar 2015a) und „Unternehmensverantwortung und Politik“ (Beschorner/Hajduk 2015) auf der Internetplattform „Ökonomenstimme“ (zugleich jeweils als Kurzbeiträge in der Neuen Züricher Zeitung publiziert) waren für den Ökonomen Rainer Maurer (2015) Anlass für eine kritische Replik. ${ }^{1}$

Rainer Maurer sieht in seinem Beitrag „Der VW-Skandal, die Wirtschaftsethik und die Wissenschaft" sehr deutlich, dass es uns in beiden Artikeln nicht nur um die Besprechung zweier interessanter Fälle („Betrug bei Volkswagen“ respektive „Positionspapier zur gesellschaftlichen Verantwortung von Unternehmen des Schweizer Bundesrates") geht, sondern damit zugleich allgemeine Fragen einer Verortung von Verantwortung awischen Individuum, Unternehmen und Staat in einer modernen, pluralen Gesellschaft verbunden sind.

Rainer Maurer stellt die These auf, dass unser Ansatz im Kern totalitäre Tendenzen habe. Unsere Argumentation ziele auf moralische Erziehungsmaßnahmen einer Gesellschaft ab, die ihren Mitgliedern ,eine ganz bestimmte Vorstellung von ,Anstand, Ehrlichkeit, Mäßigung und Gerechtigkeit" (...) vorschreibt", so der Autor. Wohin dies führe, wisse man ja aus der Geschichte. Mit Fragen der Verantwortung von Akteuren ob nun als Unternehmen oder als Person - beschäftigt sich Maurer nicht. Der VW-Fall hätte gewissermaßen abschließend gezeigt, dass „echte“ Unternehmensverantwortung in der Praxis nicht funktioniere und als dritter ,möglicher Koordinationsmechanismus zur Lösung gesellschaftlicher Probleme“" (Beschorner/Hajduk 2015) auszuschließen sei. Und auf der Ebene des Individuums hätte die Neurobiologie gezeigt: Die menschliche Willensfreiheit ist eine Illusion - und damit seien auch Fragen von individueller Verantwortung irreführend. Der Autor zieht aus diesen und weiteren Argumenten eine zentrale Konsequenz, die man wie folgt zuspitzen kann: Das ist nichts und das wird nichts mit der individuellen Verantwortung und der Verantwortung von Unternehmen. Die politische Rahmenordnung ist nicht nur der systematische, sondern auch der einzige Ort für die Umsetzung von normativen Zielen.

Diskussionen basieren mitunter darauf, dass Texte unterschiedliche Lesarten zulassen, wodurch die Intention der Verfasser und die Intentionsvermutung der Leserinnen und Leser nicht immer gut übereinstimmen müssen. Wir denken einerseits, dass dies auch in unseren beiden Texten für einige Missverständnisse gesorgt hat (und wir nehmen unser Verständnis des Textes von Maurer da nicht aus). Andererseits meinen wir aber zugleich bei Maurer und in dem ökonomischen Mainstream insgesamt durchaus gröBere „Schwierigkeiten mit der Moral“" zu erkennen, in diesem speziellen Fall: die Schwierigkeit einer theoretischen Verarbeitung der Idee moralischer Verantwortung in der ökonomischen Theorie. Dies soll Anlass dazu sein, ausgewählte Probleme ökonomischer Theoriebildung zu diskutieren.

Es überrascht kaum, dass man bei der vorherrschenden Vielzahl an Argumenten zum Verhältnis von Ökonomie und Moral schnell zu epistemologischen und ontologischen Grundsatzfragen der Mainstreamökonomik gelangt. Jene betreffen ebenso ein von uns

1 Die vollständige Diskussion, inzwischen mit weiteren Beiträgen von Rainer Maurer, findet sich hier: http://www.oekonomenstimme.org/artikel/schlagworte/\%C3\%B6konomie-moral-diskussion-2015/ (zuletzt abgerufen am 23.11.2015). 
vorgeschlagenes Alternativkonzept. Dass diese Fundamente zum Vorschein kommen, ist eine sehr begrüßenswerte Entwicklung, spielen doch solche Fragen im Alltag der Ökonomik eine eher untergeordnete Rolle. Viele Kolleginnen und Kollegen aus den Wirtschaftswissenschaften sehen sich hoffentlich nicht ungerecht dargestellt, wenn man ihnen in dieser Hinsicht drei Positionen unterstellt: Sie vertreten einen Kritischen Rationalismus, akzeptieren die Sein-Sollen-Dichotomie und sehen (vielleicht mit Ausnahmen im Bereich Behavioral Economics) die Aufgabe der Wirtschaftswissenschaften darin, sich mit Problemen der Anreizgestaltung durch Regelsetzung zu beschäftigen. Auf der Klaviatur dieser Positionen spielt auch Rainer Maurer in seiner Kritik.

Wie passt in eine solche konzeptionelle Aufstellordnung die Idee der Tugenden und die moralischen Verantwortung von Akteuren hinein?

\section{Regeln regeln Moral}

In einer Theorie, in der Menschen durch exogene Präferenzordnungen dargestellt werden, treten Probleme einer Verortung von Verantwortung auf individueller oder institutioneller Ebene aus zwei Gründen nicht auf. Zum einen stellen sich Fragen von Autonomie, Würde, Selbstbestimmung und sozialer Verhaltensprägung gar nicht, da sie im Theoriedesign ausgeschlossen sind (oder zumindest nur durch einen sehr weit gefassten Präferenzbegriff eingefangen werden können, was aber in praktischen Anwendungen nicht passiert). Und zum anderen ist bei exogenen Präferenzen die Regelebene der einzige Ort, an dem man zur Problemlösung ansetzen kann.

Von Ökonomen wird mitunter die Ansicht vertreten, ihre Beschränkung auf institutionelle Spielregeln sei eine Strategie, sich gegen opportunistisches Verhalten, dessen Existenz ja nicht geleugnet werden kann, abzusichern. Die Idee findet sich prominent bei David Hume (1963/1741: 40-42): „It is (...) a just political maxim that every man must be supposed a knave: Though at the same time, it appears somewhat strange, that a maxim should be true in politics, which is false in fact". Institutionen sollten also „schurkensicher" gestaltet werden. Wenn sie auch dann noch funktionieren, sei davon auszugehen, dass sie auch dann noch ihren Dienst tun, wenn die Menschen keine „Schurken“ sind. Damit man sich dieser Position anschließen kann, muss man aber zumindest implizit eine Annahme treffen, die darin besteht, dass es keinen Zusammenhang zwischen den Motivationen der Menschen und dem institutionellen Umfeld gibt, in dem sie leben und erzogen werden. Man kann nicht nicht-sozialisiert werden, die Frage ist nur, ob jede Form der Sozialisation dieselben Charaktereigenschaften hervorbringt. Der ökonomische Mainstream, soweit er sich der Revealed-Preference-Richtung zurechnet, kann sich zu einer solchen Annahme aber nicht äußern, da sie gegen die zugrunde liegende Idee selbigen Ansatzes, nämlich Annahmen über psychologische Prozesse zu minimieren, verstößt: Präferenzen systematisieren nur Verhalten, ohne dass wir etwas über Motive wissen können.

Weiter gilt, selbst mit einem System perfekter Regeln und perfekter Regeldurchsetzung würde sich die Frage nach der Motivation der Regelsetzer und -durchsetzer stellen. In einer Demokratie erfolgt die Regelsetzung durch politische Wahlen, und gerade für diesen Fall der Regelsetzung hat Rawls (1971) sein kohärenztheoretisches Konstrukt des fairen Schleiers des Nichtwissens entwickelt. Damit ergibt sich ein zweistufiger Prozess: Innerhalb des Regelsystems ist die moralische Rolle des Einzelnen auf die Regelbefolgung 
reduziert. Auf Ebene der Regelsetzung kommt dem Einzelnen aber die volle moralische Verantwortung bei der politischen Wahl dieser zu. Auf beiden Ebenen ist das handelnde Individuum daher als moralischer Akteur gefordert.

Die moderne Ökonomik sieht sich mit einem praktischen Problem konfrontiert: In einer Welt, in der die vollständige Internalisierung von externen Effekten durch politische Regeln gelingen könnte, müssten wir in der Tat nicht über Unternehmensverantwortung oder individuelle Moral reden. Eine solche perfekte politische Rahmenordnung gab es jedoch nie, und es gibt sie auch heute nicht. Man kann daraus folgern, dass die verbleibenden Externalitäten hinzunehmen sind, oder man zieht als Konsequenz, dass auch schon solch eine funktionalistische Sichtweise von Moral eine Nachdenken über die Verantwortung von Akteuren fordert - und zwar erst einmal gleichgültig, wie die Realisierungschancen einer praktischen Verantwortung sind.

\section{Moral Choice}

Es stellt sich die Frage, wie eine solche Sichtweise nutzbar gemacht werden kann. In Fachkreisen wird darauf sehr unterschiedlich reagiert: Eine erste Gruppe ignoriert dieses Problem, betrachtet es als nicht wesentlich oder ist der Meinung, damit sollten sich (Wirtschafts-)Ethiker beschäftigen. Eine zweite Gruppe ist engagierter, konzentriert sich aber tendenziell auf eine rhetorische, nicht argumentative Ebene (Sonntagspredigt, moralische Besserwisser, Weltverbesserer, Utopisten, Gutmenschen, etc. sind hier beliebte Metaphern zur Abwehr). Und es gibt noch diejenige Gruppe, die die Bedingungen der Möglichkeiten - nicht mehr und nicht weniger - von Verantwortungsübernahme in einer modernen Gesellschaft zu erarbeiten versucht.

Eine Dimension unserer eigenen Überlegungen zu diesem Problemkontext lässt sich in wenigen Zeilen wie folgt zusammenfassen (ausführlicher in Beschorner/Kolmar 2015b; Kolmar/Beschorner 2016): Wir sind gegenüber einer Herangehensweise an staatliche Institutionen als reine Anreizethik skeptisch und sehen die Notwendigkeit einer weitergehenden sozialwissenschaftlichen Handlungstheorie, die interpretativ angelegt ist und - besonders wichtig - Handlungstypen jenseits von Zweckrationalität kennt und diese theoretisch verarbeitet (vgl. Beschorner 2013). Für die in diesem Beitrag angesprochenen Fragen ist dabei wesentlich, dass Menschen moralisch handeln (können). Wir gehen ontologisch davon aus: Es gibt moralisches Handeln sui generis.

Es wäre gleichwohl falsch zu vermuten, wir würden aufgrund der notwendigen Unvollständigkeit politischer Rahmenordnungen von einem Extrem (nur institutionelle Regeln) in das andere (nur moralisches Handeln) verfallen. In einer komplexen arbeitsteiligen Gesellschaft kann das Individuum nicht für alle Entscheidungen, die prinzipiell moralische Signifikanz besitzen, in die Verantwortung gezogen werden. John Rawls $(1999,1971: 76)$ wies zutreffend darauf hin, dass dies zu einer hoffnungslosen Überforderung des Einzelnen führen würde. Die Setzung von Regeln reduziert Komplexität, da sich in diesem Fall die Verantwortung des Einzelnen auf die bloße Regeleinhaltung beschränkt. Aber auch hierzu ist in Abwesenheit eines perfekten Sanktionssystems der Wille erforderlich, den Regeln freiwillig zu folgen. Und wäre ein Mensch nicht eines wichtigen Teils seiner Würde beraubt, wenn er Regeln nur noch aus Angst vor Strafe einhielte? 
Ob Individuen oder Unternehmen moralisch handeln, ist abhängig von den Institutionen, innerhalb derer sie (inter-)agieren. Die Aufgabe einer institutionellen Ordnung, z.B. einer politischen Rahmenordnung, ist es damit, moralisches Handeln zu fördern und sie nicht nur über Anreizmechanismen (was Sanktionen bekanntlich einschließt) irgendwie im Zaum zu halten. Wir haben an anderer Stelle (vgl. Beschorner/Kolmar 2015b; Kolmar/Beschorner 2016) gezeigt, dass auch bei einer rein funktionalistischen Betrachtung von Moral aus dieser aristotelischen Idee der Verinnerlichung moralischer Werte Transaktionskostenvorteile über die Zeit resultieren können.

Es geht uns also insgesamt um ein geeignetes Wechselspiel von moralischem Handeln und Institutionen - in Organisationen (VW-Fall) und in der Gesellschaft (CSR-Positionspapier des Schweizer Bundesrates). In praktischer Hinsicht heißt das, dass wir weder moralisches Handeln von Mitarbeitenden als Ersatz für Organisationsstrukturen sehen noch CSR als ein Substitut für die klassische Wirtschaftspolitik.

Schon Benjamin Franklin (1787, in einem Brief an die Abbés Chalut und Arnaud) wusste: „Only a virtuous people are capable of freedom. As nations become more corrupt and vicious, they have more need of masters“. Dieses Zitat gibt eine wichtige Strömung des Liberalismus wieder, welche Moral und äußere, politische Freiheit als untrennbar aufeinander bezogen sieht. Es lässt sich aber auch auf eine Eigentümlichkeit im Kommentar von Rainer Maurer beziehen, in dem suggeriert wird, dass die Idee, den Einzelnen in die moralische Verantwortung zu nehmen, zu einer Form von Totalitarismus führe. Natürlich sind Ideen wie Umerziehung gerade im 20. Jahrhundert von totalitären Regimen auf das Unmenschlichste missbraucht worden. Aber das ist gerade nicht die Idee der Tugendethik. Vielmehr gehen diese in der Regel von der im Individuum selbst verankerten Willen zu einem gelingenden Leben aus, welches sich dann darin äussert, sich zu einem tugendhaften Menschen freiwillig zu entwickeln. Individuelle Freiheit kann auf unterschiedlichen Wegen bedroht werden. Der technokratische Glaube an eine institutionelle Lösung aller Probleme ist aus dieser Perspektive nicht Ausdruck von Toleranz und Pluralität, sondern eine Aushöhlung dieser.

\section{4. „Moral Literacy“ in der pluralen Gesellschaft}

In einer pluralen Gesellschaft sind wir uns in vielen Punkten darüber uneins, was das moralisch Gebotene ist; die meisten von uns haben ja das Gefühl, das moralisch Richtige zu wollen und oft auch zu tun. Joshua Greene (2013) nennt die damit einhergehenden Probleme auch die „Tragedy of Commonsense Morality“, die darin besteht, dass wir alle mit unseren moralischen Bauchgefühlen aufeinandertreffen und nach Wegen suchen müssen, die unterschiedlichen Legitimitätsansprüche (insbesondere auf einer globalen Ebene) miteinander in Einklang zu bringen. Weil unterschiedliche Menschen unterschiedliche Vorstellungen darüber haben, was in einer Situation z.B. gerecht ist, wäre es in der Tat anmaßend, den Begriff inhaltlich füllen zu wollen. Aus diesem Grund sind moderne Ethiken sogenannte Verfahrens- oder Prozessethiken, die sich hinsichtlich „materialer Vorgaben“ zurückhalten. Greene (2013) selbst kommt zu dem Schluss, dass eine von ihm „Deep Pragmatism“ genannte Form des Utilitarismus in der Lage ist, diese unterschiedlichen Vorstellungen zum Ausgleich zu bringen.

In pluralen Gesellschaften muss es also darum gehen, die Voraussetzungen dafür zu schaffen, dass man sich verständigt und miteinander Positionen entwickelt, die tragfähig 
sind. Dazu ist es erforderlich das eigene Bauchgefühl kritisch und unparteiisch zu hinterfragen. Tugendethische Vorstellungen sind hierzu dienlich, gehen sie doch davon aus, dass der Einzelne seine Fähigkeit, z.B. in moralischen Kategorien zu denken und zu handeln, entwickeln kann; sie sind Orientierungen zur inneren Freiheit, zu Autonomie. Die daraus resultierenden moralischen Orientierungen sind kein „Müssen“, sondern in modernen, liberalen Ethiken - ob nun kantianisch, diskurs- oder tugendethisch eingebettet - üblicherweise ein „Sollen“.

Aber es ist nicht nur die Pluralität einer Gesellschaft, die tugendethische Vorstellungen vom gelungenen Leben im Allgemeinen und vom moralisch gebotenen Leben im Besonderen attraktiv macht. Zwei andere Aspekte sind ebenso zentral. Zum einen akzeptiert eine solche Position, dass die ,richtige“ Lösung vieler Probleme nicht theoretisch antizipierbar ist, sondern sich erst situativ zeigt. Damit dies aber möglich ist, benötigen der einzelne Mensch und auch Organisationen etwas, das man „Moral Literacy“ nennen könnte, also die Fähigkeit, mit neuen Situationen auch normativ angemessen umzugehen. Die Literatur zur Praktischen Philosophie ist voll von Beispielen, anhand derer gezeigt wird, dass bestimmte universalistische Ethiken unter bestimmten Voraussetzungen Implikationen haben, die ethisch nicht überzeugen. Das liegt wohl daran, dass das Feld der Ethik keine Monokultur, sondern eine Wildnis ist, der wir nicht mit einem Prinzip gerecht werden können.

„Moral Literacy“ ist aber auch ein wichtiges Regulativ, um einen besseren Umgang mit Tendenzen der „moralischen Verschleierung“ zu fördern, die ein fester Bestandteil unserer Selbstnarrative sind. Paul Kedrosky beschreibt im New Yorker (2015), wie in komplexen Organisationen wie Volkswagen ein klar gegen moralische Standards verstoßendes Verhalten durch viele kleine, rechtfertigbare Schritte entstehen kann: „In such cultures (...) there can be a tendency to slowly and progressively create rationales that justify ever-riskier behaviors".

Auch wenn es zweifellos richtig ist, dass sich moderne Gesellschaften durch eine große Vielfalt moralischer und weltanschaulicher Positionen auszeichnen, darf darüber nicht vergessen werden, dass viele Probleme eher deutlich als undeutlich auf dem Tisch liegen. Es wird mitunter gerne so getan, als gäbe es immer moralische Differenzen, deren Auflösungen schwierig sind. Uns erscheint das nicht wirklich der Fall zu sein. So gibt es beispielsweise in Europa einen breiten Konsens zur Achtung von Menschenrechten, zu Fragen der Gleichberechtigung oder zur Notwendigkeit des Klimaschutzes. Damit sind wir schon einen Schritt weiter, denn es stellt sich bei diesen Beispielen zunehmend weniger die Frage eines „ob“ (z.B. die „Gültigkeit“ Menschenrechte zu schützen), sondern vielmehr das Problem, „wie“ normativen Anliegen Geltung verschafft werden kann. Mit Axel Honneth (2010) kann man in diesem Zusammenhang von einem „normativen Geltungsüberhang“" sprechen, den es in praktischer Hinsicht einzulösen gilt. Damit wird auch deutlich, Ethik muss nicht notwendigerweise den nächsten Anlauf zur Lösung eines „begründungstheoretischen“ Problems nehmen und sich dabei mit meist toten Philosophen herumärgern, sondern kann durchaus pragmatisch an praktischen Implementierungsfragen ansetzen (vgl. Beschorner 2015). 


\section{Schwierigkeiten mit der Normativität}

Rainer Maurer bringt in seiner Replik das „Begründungstrilemma der Ethik“ ins Spiel, das ja von Hans Albert (1968) als Münchhausen-Trilemma im Rahmen der positiven und nicht der normativen Theorie entwickelt wurde. Auch dort gilt, dass wir die Wahl zwischen den drei unschönen Alternativen Zirkelschluss, unendlicher Regress und Dogmatismus haben. De facto bleibt dabei aber nur der Dogmatismus als einzige Alternative bestehen - eben auch für die positive Wissenschaft. Jede Theorie ist notwendig von Werturteilen in diesem Sinne (als dogmatisch gesetzte erste Prinzipien) durchsetzt. Alberts vorgeschlagene Lösung des Dogmatismusproblems über das „Prinzip der kritischen Prüfung" ist selbst ein dogmatisches Prinzip auf einer anderen Begründungsebene. Denn es bedarf einer vorherigen Klärung der Frage, welche Kriterien man anlegen soll, um die kritische Prüfung durchzuführen. Diese Kriterien sind selbst nicht letztbegründet und damit dogmatisch gesetzt. Dies ist genau die Position Feyerabends (1979: 54): „Argumentieren ist für den einen Beobachter Propaganda, für den anderen das Wesen menschlicher Verständigung." Das Prinzip der kritischen Prüfung basiert auf normativen Vorstellungen, die innerhalb einer Tradition unmittelbar einleuchten können. Es sind aber stets andere Traditionen denkbar, in denen dies nicht gilt. Den „Impartial Observer“" gibt es nicht. Und selbst diese relativistische Position führt noch in eine Paradoxie oder einen ihr speziell eigenen Dogmatismus: Der Relativismus muss sich selbst als absolut setzen, um behaupten zu können, dass alles relativ ist.

Jede „Lösung“ des Dogmatismusproblems ist ein neuer Dogmatismus, der möglicherweise deshalb nicht als solcher erkannt wird, weil man so sehr Teil der Tradition ist, dass man ihre Bedingtheit nicht erkennt. Vielleicht ist es auch zielführender, den Begriff Dogma aufbauend auf Kripke (1982) durch den Begriff einer normativen Pragmatik zu ersetzen, um die pejorativen Obertöne loszuwerden und um klarzumachen, dass wir uns selbst den Grund, die Gründe, geben müssen, auf denen wir unsere wissenschaftlichen Häuser bauen können.

Aber wenn man an dieser Stelle angekommen ist, versteht man den eigentlichen grausamen Witz des Projekts der Aufklärung: Wir haben akzeptiert, dass innerbalb des Modells rationalen Argumentierens aus dem Sein kein Sollen folgt. Aber es gilt genauso, dass im Bereich der Erkenntnis jedes Sein nur aus einem Sollen folgen kann.

Wir möchten damit an dieser Stelle eine eigentlich methodische Banalität unterstreichen: Normative Fragen entstehen für sozialwissenschaftliche Theorien jedweder Art nicht nur im Anschluss an die durchgeführten Untersuchungen ${ }^{2}$, sondern sie beginnen bereits mit der Frage „was man vorne reinsteckt“, z.B. in Form von Verhaltensannahmen über Akteure. Wir können uns der normativen Prämissen bewusst sein oder nicht, diese offenlegen oder nicht; in jedem Fall gilt: Theorien fußen immer auf normativen Annahmen. Die Tatsache, dass auch positive Theorien begründungstheoretische Probleme haben, macht es für die normativen Theorien nicht besser, zeigt aber, dass der Unterschied zwischen positiv und normativ nicht so groß ist, wie vielleicht vermutet. 
Die Auseinandersetzung mit der Frage, wie man in einer pluralen Gesellschaft mit heterogenen moralischen Positionen umgehen kann, ist dabei ferner zunächst einmal von der Frage zu trennen, welchen Wahrheitswert ethische Prinzipien haben. Zur zweiten Frage ist seit David Humes (1739, 1978) Feststellung eines Sein-Sollen-Fehlschlusses viel geschrieben worden. Es ist dabei interessant zu vermerken, dass selbst George Edward Moore (1903), auf den der oft missverstandene Begriff des naturalistischen Fehlschlusses zurückgeht, nach Christine Korsgaard (1992) eine spezielle Form des ethischen Realismus vertreten hat. Die Einsicht, dass innerhalb eines ontologisch-epistemischen Systems naturalistische Fehlschlüsse möglich sind, bedeutet daher noch nicht, die Wahrheitsfähigkeit normativer Aussagen zurückzuweisen. Selbst David Hume ging von der Möglichkeit moralischer Erkenntnis aus. Sein Argument des SeinSollen-Fehlschlusses richtete sich gegen eine Rechtfertigung moralischer Verpflichtungen im Sinne des Realismus. Für ihn resultierte die Verpflichtung aus der Natur des Menschen als soziales Wesen; Christine Korsgaard (1992) nennt dies das Verfahren der reflexiven Billigung.

Wenn wir nun fragen, woher eine bestimmte ethische Position ihre Verpflichtungswirkung für den Einzelnen bezieht, kennen wir wenigstens zwei Argumentationsweisen, die dieses begründungstheoretische Vakuum zu füllen versuchen. Die eine geht auf Immanuel Kant zurück und basiert auf der Idee, dass der Einzelne gebunden ist an die Praktische Vernunft, so dass er das moralisch Gebotene aus Pflicht verrichtet. Und die andere ist durch die Tugendethik gegeben, die in gewisser Hinsicht die Kantsche moralische Pflicht verkörpern möchte, so dass der tugendhafte Mensch Pflicht und Neigung zur Deckung bringt. Ein nicht weiter eingebetteter Verweis auf die exklusive Rolle von Institutionen zur Lösung normativer Fragen löst das Begründungsproblem, indem es dieses ignoriert.

\section{Praxis und Kultur}

Rainer Maurer stößt sich an unserer Kritik an einer naturwissenschaftlich-orientierten Ökonomik und fragt, wie „eine moderne Ethik ohne Berücksichtigung erfahrungswissenschaftlicher und damit auch naturwissenschaftlicher Erkenntnisse auskommen kann“. Es ist aus unserer Sicht völlig zutreffend, dass eine jede Ethik die konkreten Lebens- und Systemwirklichkeiten ihres jeweiligen Reflexionsfeldes nicht nur zur Kenntnis nimmt, sondern diese auch theoretisch verarbeitet.

Es wurde dazu an anderer Stelle (vgl. Beschorner 2013; 2015) formuliert, dass moralische Probleme in der Praxis nicht nur Anlass, sondern methodischen Ausgangspunkt für eine Ethik darstellen sollten. Wir bezweifeln nicht, dass auch „,naturwissenschaftliche Erkenntnisse“ hierzu beitragen können. Dies jedoch angesichts reichlich kulturell aufgeladener Lebenswelten und systemischer Wertsphären auf naturwissenschaftliche Phänomene zu beschränken, erscheint uns unzureichend.

\section{Neurowissenschaften und der „(un)freie Wille“?}

Rainer Maurer erachtet „erfahrungswissenschaftliche Erkenntnisse“ aus den Neurowissenschaften für besonders relevant und hält dazu mit Blick auf moralische Fragen noch eine Pointe bereit: Die Ultima Ratio, das Individuum aus der moralischen Verantwortung zu entlassen, in der wir es gerne stärker sähen, besteht für ihn darin, ihm den freien 
Willen abzusprechen. Aber wenn Menschen keinen freien Willen haben, dann kann man sie nach herrschender Auffassung auch nicht für ihr Verhalten moralisch verantwortlich machen. Was ist von Rainer Mauers Argument zu halten?

Zunächst sollte man sich bewusst machen, dass eine solche gesellschaftstheoretische Konzeption verdächtig wenig nach liberalem Staat und Demokratie aussieht, sondern vielmehr nach Skinnerschem Behaviorismus. Aber mehr zum Punkt. Die Frage nach dem freien Willen berührt Grundsatzfragen, die wichtig sind. In der philosophischen Debatte zu diesem Thema ist die aus unserer Sicht herrschende Meinung, dass die Interpretation neurowissenschaftlicher Ergebnisse als Evidenz für oder gegen Willensfreiheit einen Kategorienfehler darstellt (vgl. Habermas 2004; 2006).

Um diesen zu verstehen, muss man zwischen Gehirn und Geist unterscheiden (vgl. Dietrich/List 2015). Gehirn bezieht sich dabei auf die physischen Mechanismen, die einer Beobachtung „,von außen“ zugänglich sind. Beobachtung des Gehirns impliziert eine Dritte-Person-Perspektive. Geist bezieht sich hingegen auf das subjektive Erleben des Menschen. Es handelt sich um eine Erste-Person-Perspektive. Willens- oder Handlungsfreiheit erschließen sich uns aus einer solchen Erste-Person-Perspektive; in diesem Bereich erleben wir uns als frei, glücklich, verzweifelt, oder was auch immer. Phänomenologisch bedeutet das, dass wir als Individuen in der Erste-Person-Perspektive existieren $^{3}$. Die Frage ist nun, inwieweit die Verbundenheit der Erste- mit der Dritte-Person-Perspektive und der (angenommene) naturwissenschaftliche Determinismus bei der Beschreibung des Gehirns dazu Anlass gibt, unser Erleben von Willensfreiheit als Illusion zu verwerfen.

Um die Frage nach der Willensfreiheit stellen zu können, muss zunächst geklärt werden, was man darunter versteht. Tugendhat (2007: 57ff) unterscheidet zwischen Handlungsfreiheit und Willensfreiheit und argumentiert, dass die Frage, ob ich meine Hand heben kann, wenn ich will (vgl. Libet-Experiment, z.B. in Libet 1999), nichts mit Willens-, sondern mit Handlungsfreiheit zu tun hat. „Bei dieser gibt es gar keinen Grund, am Verursachtsein des Wollens Anstoß zu nehmen“. Aufbauend auf Kant und Locke argumentiert Tugendhat (ebd.), dass „Willensfreiheit das Vermögen ist, nicht nur von unmittelbar empfundenen Motiven in seinem Handeln bestimmt zu sein, sondern nach Gründen zu handeln". Anders als bei der Handlungsfreiheit erscheint eine Person nicht nur frei, etwas zu tun, sondern frei, ihr Wollen zu bestimmen. Dies setzt ein handelndes Ich, eine Konzeption von Zukunft und ein normatives Konzept voraus, welches die unmittelbaren Handlungsimpulse mit der Vorstellung des langfristig Guten abgleicht.

Die Begriffspaare „Willensfreiheit“ und „Zwang“ auf der einen und „Determinismus“ und „Indeterminismus“ auf der anderen Seite gehören unterschiedlichen Beschreibungsebenen an. Eine Vermischung dieser bedeutet einen Kategorienfehler (vgl. Bieri 2006; Habermas 2004; 2006). Determinismus und Willensfreiheit sind daher vereinbar, weil beide Beschreibungsebenen nichtreduzierbar nebeneinander bestehen. Tugendhat (2007: 57ff) argumentiert für eine solche Irreduzierbarkeit, indem er ausführt, dass die Ich-Perspektive nicht in eine Dritte-Person-Perspektive übersetzbar ist: „Hier drängt sich nun aber (...) auf, dass nicht zu sehen ist, wie es überhaupt möglich sein soll, das 
Bewusstsein, dass etwas von mir abhängt, (...) in eine objektive Sprache zu übersetzen, in der das Wort «ich» nicht vorkommt“".

Wir bezweifeln nicht, dass aus den Neurowissenschaften interessante Einsichten für die Sozialwissenschaften (z.B. die Ökonomik) resultieren und weiterhin resultieren werden. Aus diesen Ergebnissen die Beschränkung des freien Willens abzuleiten, erscheint uns jedoch so nicht möglich zu sein.

\section{Metaphysik - immer}

Rainer Maurer entwickelt Teile seiner Kritik auf Basis des Falsifizierbarkeitskriteriums des Kritischen Rationalismus. Um das Argument spannend zu machen, akzeptieren wir die Schlussfolgerung, unsere Aussage sei nicht falsifizierbar und daher metaphysischer Kokolores. Dann sind zwei Dinge anzumerken.

Zum einen gilt dieselbe Schlussfolgerung natürlich auch für die umgekehrte Hypothese, dass eine Gesellschaft, die alle Probleme durch die Schaffung institutioneller Regeln lösen will, die Probleme in den Griff bekommen kann und dabei liberalen (in einem bestimmten, näher zu bestimmenden Sinn) Prinzipien treu bleibt; sie ist ebensolcher metaphysischer Kokolores. Jeder Versuch, das gesellschaftliche Zusammenleben auf bestimmten Prinzipien zu fußen, basiert notwendig auf in diesem Sinne metaphysischen Voraussetzungen, ob wir uns derer bewusst sind oder nicht. Die Zukunft ist in einem Sinne offen, dass wir über das Gelingen unserer Anstrengungen nur spekulieren können.

Was ist zum Verfahren der Falsifizierbarkeit zu sagen? Die meisten (alle?) wissenschaftlichen Falsifikationsversuche konfrontieren wissenschaftliche Hypothesen nicht mit „der Realität“, sondern mit Hypothesen anderer „Wirklichkeiten“, die innerhalb einer anderen Theorie abgeleitet wurden. Falsifikation bedeutet dann nicht mehr und nicht weniger, als dass beide Theorien miteinander unverträglich sind. Eine Korrespondenztheorie der Sprache (also eine Prämisse der Art, dass die Ordnung der Welt der Ordnung der Sprache entspricht und durch sie abgebildet werden kann) ist philosophisch heftig umstritten (Marian 2015). Wir können sie aus pragmatischen Gründen vielleicht nutzen, müssen uns aber darüber bewusst bleiben, auf welchen Prämissen sie basiert ${ }^{4}$.

\section{Fin}

Die Ökonomik tut sich schwer mit der Moral. Will sie diese Schwierigkeiten beheben, so erscheinen Grundsatzdiskussionen notwendig, die eine stärkere Reflexion des philosophischen Voraussetzungsreichtums ökonomischer Theorien zum Ziel haben. Es hat uns durchaus erstaunt, dass gerade die Idee des notwendigen Ineinandergreifens von Verantwortung und freiheitlicher Gesellschaft als (versteckter) Angriff auf eben diese gewertet wird. Ist eine Gesellschaft, die ohne Verantwortung von Akteuren auskommen und alles durch staatliche Institutionen regeln will, tatsächlich frei?

$4 \quad$ Siehe hierzu auch das sogenannte Unterbestimmtheits- oder Duhem-Quine-Problem (Quine 1979). 


\section{Literatur}

Albert, H. (1968): Traktat über kritische Vernunft, Tübingen: Mohr Siebeck.

Beschorner, T. (2013): Kulturalistische Wirtschaftsethik: Grundzüge einer Theorie der Anwendung, in: Zeitschrift für Wirtschafts- und Unternehmensethik, Jg. 14/Heft 3, 346-372.

Beschorner, T. (2015): Erkundungen zu einem noch nicht gefundenen Ort des „moral point of view“, in: Beschorner, T./Ulrich, P./Wettstein, F. (Hrsg.): St. Galler Wirtschaftsethik. Programmatik - Positionen - Perspektiven, Marburg: Metropolis-Verlag, 305-336.

Beschorner, T./Hajduk, T. (2015): Unternehmensverantwortung und Politik, in: Ökonomenstimme vom 21.09.2015. Link: http:/ /www.oekonomenstimme.org/artikel/2015/09/ unternehmensverantwortung-und-politik/ (zuletzt abgerufen am 02.11.2015).

Beschorner, T./Kolmar, M. (2015a): Die Ökonomik und ihre Schwierigkeiten mit der Moral, in: Ökonomenstimme vom 22.10.2015. Link: http://www.oekonomenstimme.org/ artikel/2015/10/die-oekonomik-und-ihre-schwierigkeiten-mit-der-moral/ (zuletzt abgerufen am 02.11.2015).

Beschorner, T./Kolmar, M. (2015b): Moral Capabilities and Institutional Innovation - An Extended Transaction Cost Approach, in: Enderle, G. (Hrsg.): Ethical Innovation in Business and the Economy, Cheltenham: Edward Elgar Publishing (im Erscheinen).

Bieri, P. (2006): Untergräbt die Regie des Gehirns die Freiheit des Willens? In: Heinze, M./Fuchs, T./Reischies, F. M. (Hrsg.): Willensfreiheit - eine Illusion?, 35-48.

Dietrich, F./List, C. (2015): Mentalism versus Behaviourism in Economics: A Philosophy-of-Science Perspective, Working Paper. http://dx.doi.org/10.2139/ssrn.2033165 (zuletzt abgerufen am 02.11.2015).

Greene, J. (2013): Moral Tribes: Emotion, Reason, and the Gap Between Us and Them, London: Penguin Press.

Feyerabend, P. (1979): Erkenntnis für freie Menschen. Frankfurt/M.

Habermas, J. (2004): Freiheit und Determinismus, in: Deutsche Zeitschrift für Philosophie, Jg. 52/Heft 6, 871-890.

Habermas, J. (2006): Das Sprachspiel verantwortlicher Urheberschaft und das Problem der Willensfreiheit: Wie lässt sich der epistemische Dualismus mit einem ontologischen Monismus versöhnen?, in: Deutsche Zeitschrift für Philosophie, Jg. 54/Heft 5, 669-707.

Hausman, D. (1998): Rationality and Knavery, in: Leinfellner, W./Köhler, E. (Hrsg.): Game Theory, Experience, Rationality; Foundations of Social Sciences; Economics and Ethics: In Honor of John C. Harsanyi, Dordrecht: Kluwer, 67-79.

Honneth, A. (2010): Das Ich im Wir. Studien zur Anerkennungstheorie, Berlin: Suhrkamp.

Hume, D. (1963/1741): Of the Independency of Parliament, in: Hume, D.: Essays Moral, Political, and Literary, Oxford: Oxford University Press.

Hume, D. (1739, 1978): A Treatise of Human Nature, London: John Noon. Oxford: Clarendon Press

Kabneman, D. (2011): Thinking Fast and Slow, London: Macmillan.

Kedrosky, P. (2015): An Engeneering Theory of the Volkswagen Scandal, The New Yorker. Link: http://www.newyorker.com/contributors/paul-kedrosky (zuletzt abgerufen am 02.11.2015). 
Kolmar, M./Beschorner, T. (2016): Locating Responsibility - An Extended Transaction Cost Approach, in: Zeitschrift für Wirtschafts- und Unternehmensethik, Jg. 17/Heft 1 (im Erscheinen).

Korsgaard, C. (1992): The Sources of Normativity - The Tanner Lectures on Human Values, Cambridge: Cambridge University Press.

Kripke, S. (1982). Wittgenstein on Rules and Private Language. Harvard University Press.

Libet, B. (1999): Do we have a free will? In: Journal of Consciousness Studies, 5.

Marian, D. (2015): The Correspondence Theory of Truth, The Stanford Encyclopedia of Philosophy (Fall 2015 Edition), Edward N. Zalta (ed.), Link: http://plato.stanford.edu/archives/fall2015/entries/truth-correspondence/ (zuletzt abgerufen am 02.11.2015).

Maurer, R. (2015): Der VW-Skandal, die Wirtschaftsethik und die Wissenschaft, in: Ökonomenstimme vom 13.10.2015. Link: http://www.oekonomenstimme.org/artikel /2015/10/der-vw-skandal-die-wirtschaftsethik-und-die-wissenschaft/ (zuletzt abgerufen am 02.11.2015).

Moore, G. E. (1903): Principia Ethica, Cambridge: Cambridge University Press.

Quine, W. v. O. (1979): From a Logical Point of View. Nine Logico-Philosophical Essays, Cambridge, Mass.: Harvard University Press.

Rawls, J. (1999, 1971): A Theory of Justice, Revised Edition Cambridge, Mass.: Harvard University Press.

Tugendhat, E. (2007): Anthropologie statt Metaphysik, München: Beck. 